Psychiatrie de l'enfant, L, 1/2007, p. 179 - 203.

\title{
RECHERCHE CLINIQUE
}

\author{
Classification Diagnostique 0-3 ans \\ Recherche sémiologique \\ Troubles de la Régulation \\ Hypersensible \\ Hyposensible/Sous-réactif \\ Cherchant des stimulations sensorielles et/ou Impulsif \\ Traitement de l'information visuo-spatiale \\ Traitement de l'information auditivo-verbale \\ Perception des affects \\ Organisation émotionnelle
}

\section{CLASSIFICATION DIAGNOSTIQUE 0-3 ANS : RECHERCHE SÉMIOLOGIQUE SUR LES TROUBLES DE LA RÉGULATION}

\author{
Jean-Marc SCHOLL ${ }^{1}$
}

\section{CLASSIFICATION DIAGNOSTIQUE 0-3 ANS : RECHERCHE SÉMIOLOGIQUE SUR LES TROUBLES DE LA RÉGULATION}

Dans la Classification Diagnostique 0-3 ans, les Troubles de la Régulation des stimuli sensoriels se réfèrent aux difficultés de ces enfants à réguler leurs émotions, leurs comportements et leurs habilités motrices en réponse aux stimulations sensorielles. Cet article présente une recherche qui a pour finalité de mieux préciser la sémiologie de micro-signes - repérables dans la clinique et l'anamnèse - de Troubles de la Régulation. On donne ici les résultats d'une phase préliminaire de cette recherche. Elle consiste en une microanalyse qualitative de 90 situations cliniques de Trouble de la Régulation d'enfants de 7 mois à 5 ans. Une attention particulière est donnée au traitement de l'information visuo-spatiale, auditivo-verbale, à la perception des affects, aux modalités de leurs expressions, au "niveau énergétique", à l'organisation de la sphère buccale, à l'hyper ou l'hyposensibilité de chacun des canaux sensoriels et à tout symptôme qui est en lien à un support constitutionnel. Cette phase préliminaire a permis d'élaborer un questionnaire qui est à la base de la deuxième phase de la recherche : en comparant les résultats de ce questionnaire anamnestique, entre un groupe témoin et un groupe d'enfants présentant un diagnostic de Trouble de la Régulation, nous pourrons mieux préciser les signes spécifiques de ces derniers et leur fréquence. Cette étude voudrait contribuer à mieux décrire les tableaux cliniques de ces enfants et, à enrichir les échanges pluridisciplinaires et les pratiques de psychothérapie.

\section{DIAGNOSTIC CLASSIFICATION ZERO TO THREE : SEMIOLOGICAL RESEARCH ON REGULATION DISORDERS}

In the Diagnostic Classification Zero to Three, Regulation Disorders of Sensory Processing refer to children's difficulties in regulating emotions, behaviours and motor skills in response to sensory stimulation. This article presents a research program that aims to specify more accurately the semiology of micro-signs -

\footnotetext{
1. Pédopsychiatre. Service de Santé Mentale (Verviers, Belgique). Recherche réalisée sous la tutelle de l'Université de Liège (Professeurs Jean-Marie Gauthier, Marc Ansseau et Francis Pérée). J'adresse mes remerciements au Dr Paule Philippe pour les échanges cliniques qui ont alimenté ce travail, de même à chacun des membres du S.S.M. de Verviers.
} 
identifiable in the clinical symptoms and in anamnesis - of Regulation Disorders. We give here the results of a preliminary stage of research, which consists of a qualitative microanalysis of 90 Regulation Disorder cases in children between 7 months and 5 years old. We give particular attention to visual-spatial processing, auditoryverbal processing, the perception of the affects, the modalities of their expression, the "energy level" of the child, the organisation of the buccal sphere, the hyper- or hyposensitivity of each sensory channel, and all symptoms having a connection to some constitutional platform. This preliminary stage serves as the basis of a questionnaire which is at heart of the second step of the research. We will further clarify the specific signs of Regulation Disorders and their frequency, comparing the results of this anamnestic questionnaire using a control sample and a sample of children with Regulation Disorders. This study aims to contribute to a more accurate description of the clinical pictures of these children and to enrich multidisciplinary exchanges and psychotherapeutic practices.

\section{CLASIFICACIÓN DIAGNÓSTICA DE 0 A 3 AÑOS: INVESTÍGACIÓN SEMIOLÓGICA SOBRE EL TRASTORNO DE REGULACIÓN.}

En la clasificación Diagnóstica de 0 a 3 años, los Trastornos de la Regulación de los estímulos sensoriales se refieren a las dificultades de los niños para regular sus emociones, sus comportamientos y sus habilidades motrices de respuesta a los estímulos sensoriales. Este artículo presenta una investigación que tiene por finalidad precisar mejor la semiología de los micro-signos-identificables en los síntomas clínicos y la anamnesis.- de los Trastornos de Regulación. Presentamos aquí los resultados de una fase preliminar de esta investigación. Esta consiste en un microanálisis cualitativo de 90 situaciones clínicas del Trastorno de la Regulación en niños de 7 meses a 5 años. Se ha dado una atención particular al tratamiento de la información viso-espacial, auditivo verbal, a la percepción de los afectos, a las modalidades de sus expresiones, al "nivel energético", a la organización de la esfera bucal, a la hiper o hiposensibilidad de cada uno de los canales sensoriales y a todo síntoma que se encuentra ligado a un soporte constitucional. Esta fase preliminar ha permitido elaborar un cuestionario que se encuentra en la base de la segunda parte de la investigación : comparando los resultados de este cuestionario de anamnesis, entre un grupo de control y un grupo de niños que presentan un diagnóstico del Trastorno de la Regulación podremos precisar mejor los signos específicos de éstos últimos y su frecuencia. Este estudio querría contribuir a describir mejor los cuadros clínicos de estos niños y de enriquecer los intercambios pluridisciplinares y las prácticas de psicoterapia.

\section{CIRCONSTANCES À L'INITIATIVE DE LA RECHERCHE}

Le Conseil Régional de la Région Wallonne, à l'initiative du professeur Gauthier (Université de Liège), a sollicité les Services de Santé Mentale qui prennent en traitement des enfants de 0 à 3 ans, afin de créer un groupe de travail expérimental d'utilisation de la Classification "0 to 3". Depuis janvier 2002, des rencontres régulières de 20 Centres ont permis de se familiariser avec cette classification et d'en découvrir la richesse clinique. La grande fréquence d'enfants de 0 à 6 ans présentant des Troubles de la Régulation a incité l'auteur de cet article à créer un programme de recherche. Cet article en présente les premiers résultats et les perspectives ultérieures.

\section{LA CLASSIFICATION 0-3 ANS ET LES TROUBLES DE LA RÉGULATION}

\section{A) La Classification 0-3 ans}

La Classification Diagnostique "Zero to three" fut publiée aux USA en 1994 (Zero to three, 1994). Elle est le résultat d'un groupe de travail multidisciplinaire créé en 1987 et constitué de cliniciens et de chercheurs provenant de centres de l'enfance des USA, auxquels se sont joints des centres du Canada et d'Europe. Cette nomenclature veut être une simple photographie de l'instant présent, toujours susceptible de modifications et d'évolution. La classification est multi-axiale, relationnelle, souple, dynamique pour la connaissance et le traitement des nourrissons, des jeunes enfants et de leur famille (Thomas et Harmon, 1998). Elle a rencontré une 
reconnaissance internationale et elle est traduite ou en cours de traduction en 14 langues. Des études de validité démontrent sa fiabilité (Guedeney et al., 2002). Une révision de cette classification est parue en 2005 (DC:0-3R).

Dans cette nomenclature multi-axiale les Troubles de la Régulation du traitement des stimuli sensoriels apparaissent dans l'axe I consacré aux diagnostics primaires de l'enfant. Dans notre pratique, cette nomenclature - et particulièrement pour ce qui se rapporte aux Troubles de la Régulation - est utilisable jusqu'à l'âge de 6 ans et pourrait donc s'appeler : "0 to 6".

\section{B) Description générale des Troubles de la Régulation}

«Les Troubles de la Régulation sont caractérisés par les difficultés du bébé ou du jeune enfant à réguler ses comportements et ses processus physiologiques sensoriels, d'attention, moteurs ou émotionnels et à maintenir un état de calme, de vigilance ou un état émotionnel positif »(Classification 0-3 ans, 1998, p.41).

Les processus physiologiques impliqués se rapportent à des difficultés de traitement de l'information sensorielle, sensori-motrice ou à des difficultés de son organisation, telles que des difficultés à réguler, à intégrer et à répondre à divers types de sensations (y compris auditives et visuelles) et à divers types d'affects. Ceci influence l'adaptation journalière de l'enfant, ses interactions et ses relations. Sa sensibilité peut aussi varier tout au long de la journée. Le plus souvent, l'apport sensoriel tend à se cumuler, si bien qu'il est possible qu'un enfant ne soit pas gêné initialement mais rencontre des difficultés notables à la fin de la journée. En outre, la réaction aux stimuli sensoriels est influencée par le niveau de base de l'éveil. Si l'enfant est stressé ou fatigué, il est probable qu'il faudra moins d'apports sensoriels pour déclencher une réaction hypersensible.

L'organisation émotionnelle est prise en compte. Elle comprend la tonalité émotionnelle prédominante (par exemple, calme, déprimé ou heureux), l'éventail des affects (par exemple, large ou restreint), le degré de modulation exprimé (par exemple, le bébé passe brusquement d'un calme complet à des hurlements frénétiques) et la capacité d'utiliser et d'organiser les affects comme faisant partie des relations et de l'interaction avec les autres (par exemple un type de comportements de style évitant, des comportements d'opposition, d'agrippement et d'exigences,...).

Les parents peuvent constater que l'enfant est facilement bouleversé ou se met vite en colère, et qu'il a des difficultés à s'adapter aux changements.

\section{C) Diagnostic des Troubles de la Régulation}

La nouvelle Classification «Diagnostic Classification of Mental Health and Developmental Disorders of Infancy and Early Childhood: REVISED EDITION »: « DC:0-3R » (Zero to three, 2005) donne trois modalités de Troubles de la Régulation du traitement des stimuli sensoriels :

1. Hypersensible

2. Hyposensible/Sous-réactif

3. Recherchant des stimulations sensorielles et/ou Impulsif

La modalité Hypersensible est elle-même subdivisée en deux types : Type A: Craintif/Prudent

Type B: Opposant/Provoquant

Ces Troubles de la Régulation sont inclus dans l'axe I des diagnostics primaires, dans la catégorie 400 qui en précise plusieurs tableaux cliniques avec les références suivantes :

Axe I

400. Trouble de la Régulation du traitement des stimuli sensoriels 410 : Hypersensible

411 : Type A: Craintif/Prudent

412 : Type B: Opposant/Provoquant

420 : Hyposensible/Sous-réactif

430 : Recherchant des stimulations sensorielles et/ou Impulsif

Chacun des tableaux cliniques est décrit en trois volets :

1. Les patterns de la réactivité sensorielle. Ils décrivent les difficultés du traitement des stimuli sensoriels.

2. Les patterns moteurs. Ils décrivent les difficultés motrices.

3. Les "patterns d'attitudes". Ils décrivent les attitudes et comportements spécifiques.

Pour chaque modalité de Trouble de la Régulation du traitement des stimuli sensoriels des réactions spécifiques à ces stimuli apparaissent chez les enfants. Les nourrissons et les enfants Hypersensibles aux stimuli sensoriels les expérimentent comme désagréables et ils les évitent. Les enfants Hyposensibles/Sous-Réactifs ont besoin d'une forte intensité d'entrée sensorielle avant de pouvoir y répondre. Les enfants Recherchant les stimulations sensorielles et/ou Impulsifs cherchent activement à satisfaire leurs besoins d'entrées sensorielles de haut niveau. 


\section{D) Les diagnostics différentiels}

La Classification donne les diagnostics différentiels et les comorbidités possibles aux Troubles de la Régulation.

Les situations cliniques des Troubles de la Régulation vont des plus légères aux plus importantes pour arriver à la frontière des "Troubles touchant de Multiples Domaines du Développement" (catégorie 700, faisant partie des diagnostics primaires de l'axe I). Ces derniers, pour les formes les plus sévères, se rapprochent du spectre autistique. Dans ces situations frontières, c'est le sens clinique qui permet de différencier "un Trouble touchant de Multiples Domaines du Développement avec des traits de Troubles de la Régulation" et "un Trouble sévère de la Régulation avec des caractéristiques mixtes". Ces deux états seront très sensibles à des situations de stress important de la vie. La différenciation sera fonction de l'importance des difficultés liées à la relation, du degré des troubles du langage et de la communication, et de la plus ou moins bonne réponse initiale aux interventions thérapeutiques. (De même, dans les Troubles touchant de Multiples Domaines du Développement, la difficulté relationnelle n'est pas considérée comme un déficit permanent et relativement figé mais comme susceptible de modifications et d'évolution) (Classification 0-3 ans, 1998, p.128).

\section{OBJECTIF DE LA RECHERCHE : DONNER UN MEILLEUR SENS CLINIQUE DES TROUBLES DE LA RÉGULATION ET NOTAMMENT DE LA COMPOSANTE CONSTITUTIONNELLE}

\section{A) Intérêts de cette première étape de la recherche}

« De nombreux troubles de l'attention, de l'expression émotionnelle, de la motricité, de la sensorialité, de la maîtrise du comportement et du langage qui, traditionnellement, étaient considérés comme des difficultés en elles-mêmes peuvent, chez certains enfants, faire partie d'un Trouble de la Régulation plus important » (Classification 0-3 ans, 1998, p.42). Une meilleure compréhension de difficultés sous-jacentes permettrait de rattacher ces éléments cliniques élémentaires à des entités plus larges "d'inter-dysfonctionnements" en rapport à des tableaux cliniques.

«Les cliniciens ont utilisé les termes généraux, tels que "enfant trop sensible", "tempérament difficile" ou "réactif" pour décrire des modèles sensoriels, moteurs et intégratifs dont on présume qu'ils sont d'origine "constitutionnelle" ou "d'ordre biologique", mais sans décrire spécifiquement le cheminement sensoriel ou les fonctions motrices impliquées. Il est de plus en plus évident que les patterns constitutionnels et ceux de la maturation précoce contribuent aux difficultés de ces bébés, mais il est aussi reconnu que les différents modes de soins précoces peuvent exercer une influence considérable sur la façon dont se développent les patterns constitutionnels et maturationnels et sur la façon dont ils deviennent partie intégrante de l'élaboration de la personnalité de l'enfant. » (Classification 0-3 ans, 1998, p.42).

Cette étude pourrait, à partir de la symptomatologie, contribuer à un meilleur discernement -- dans un continuum -- entre la part constitutionnelle, la part psychologique et la part relationnelle intervenant dans les difficultés de l'enfant.

À mesure que l'intérêt vis-à-vis de ces enfants augmente, il est important de systématiser la description des patterns sensoriels, moteurs et intégratifs dont on suppose l'implication.

«Les Troubles de la Régulation du traitement des stimuli sensoriels sont constitutionnellement déterminés par les réponses de ces enfants aux stimuli sensoriels. Les stimuli sensoriels comprennent le toucher, la vue, les bruits, le goût, l'odorat, les sensations de mouvements dans l'espace, et la conscience de la position du corps dans l'espace. Chaque enfant a une modalité spécifique pour répondre aux stimuli sensoriels de l'environnement. Certains enfants ont des difficultés $\left(1^{\circ}\right)$ au niveau du traitement des stimuli sensoriels entrant et $\left(2^{\circ}\right)$ au niveau de la régulation des réponses qu'ils apportent à ces stimuli. Ces difficultés à traiter les stimuli et à réguler leurs réactions peuvent interférer avec l'ensemble du développement social et émotionnel de l'enfant et avec ses habilités motrices, plus particulièrement avec l'habilité de l'enfant à participer aux activités appropriées à son âge. » (Zero to three, 2005, p.28)

Repérer et observer ces difficultés du traitement des stimuli sensoriels et de la régulation des réponses à ces stimuli peut déconcerter le clinicien car il est peu habitué, dans la pratique de psychothérapie, à rechercher ces difficultés et encore moins à les lire en termes constitutionnels "neurophysiologiques". Cette recherche voudrait développer le sens clinique et la sémiologie de ces difficultés constitutionnelles et la fréquence de leur apparition. On cherchera à encore mieux préciser les tableaux cliniques de la Classification.

Cette clinique et cette recherche sont à la convergence de l'intérêt tant des pédopsychiatres, psychologues, psychomotriciens, ergothérapeutes, thérapeutes du développement, que des neuropédiatres confrontés à des enfants de 0 à 5 ans, de plus, elles peuvent enrichir la pluridisciplinarité et les pratiques de la psychothérapie. 


\section{B) Méthode pour la partie préliminaire de la recherche}

1. Analyse du matériel clinique - anamnestique et sémiologique - de 90 enfants ayant un diagnostic de Troubles de la Régulation du traitement des stimuli sensoriels ou d'un "Trouble sévère de la Régulation avec des caractéristiques mixtes avec un Trouble touchant de Multiples Domaines du Développement". Lors du premier rendez-vous ces enfants avaient entre 7 mois et 5 ans. $70 \%$ d'entre eux ont toujours un suivi.

2. Pour ces enfants : recherche sémiologique de micro-signes repérables de ces Troubles de la Régulation. Nous appellerons ces micro-signes des "dérégulations".

3. De même, recherche des éléments anamnestiques correspondant à ces "dérégulations".

4. À partir de ces données préalables, nous élaborons un questionnaire qui cherche à mettre en évidence les Troubles de la Régulation chez les enfants de 0 à 6 ans. Ce questionnaire sera utilisé pour une étape ultérieure de la recherche ; il sera rempli par des parents d'enfants présentant des Troubles de la Régulation (cf. infra).

\section{RÉSULTATS CLINIQUES PRÉLIMINAIRES ACTUELS}

\section{A) Les éléments anamnestiques}

Nous ne pouvons reprendre tous les résultats qui à eux seuls feraient l'objet d'un article. Mentionnons simplement que cette anamnèse comprend : les perceptions extéroceptives et intéroceptives, l'humeur, l'expressivité, le tempérament, les attitudes (têtu-obstiné, peureux,...), la perception des affects, la capacité de les exprimer, leurs modulations, l'image de soi, l'évaluation du temps, la capacité à être seul, la clinique du sommeil, l'alimentation, l'attention (selon différents canaux sensoriels), l'hyperactivité, l'impulsivité, le niveau énergétique, l'aspect qualitatif des colères, des "débordements", la sphère O.R.L., l'évolution développementale, la scolarité, les antécédents médicaux,...

\section{B) La sémiologie des "dérégulations"}

Ces "dérégulations" comprennent les difficultés à traiter les stimuli et à réguler les réponses à ces stimuli. La Classification cite parmi elles : sur ou sous-réactivité à des bruits, à des lumières, aux odeurs, à la température, à la douleur, au contact, au toucher, aux sensations gravitationnelles, de la sensibilité buccale, et régulation de la tonicité et de la stabilité musculaire, de l'organisation motrice, de la motricité fine, de l'articulation des mots, de l'attention et la concentration, du traitement de l'information auditivo-verbale, du traitement de l'information visuo-spatiale (Classification 0-3 ans, 1998, p.43).

Le clinicien peut rester déconcerté dans cette recherche. Voici des exemples ponctuels concrets pour aider à donner un meilleur sens clinique.

\section{Avertissement}

Les exemples suivants sont issus de l'analyse des 90 situations cliniques de notre échantillon de départ. Ils ne sont mentionnés que rapidement sous forme d'items et en dehors de la présentation clinique d'ensemble. Le plus souvent, chaque item pris isolément n'a pas de valeur et est non pertinent s'il n'est pas rapporté au tableau clinique de la situation correspondante. Ces exemples ont toutefois le mérite de développer un préliminaire à la recherche sémiologique. Ici nous regroupons spécifiquement des items se rapportant à un niveau de lecture constitutionnelle. Bien sûr, il ne s'agit pas de limiter la lecture d'une situation clinique à ce seul niveau, ce serait réducteur. La réalité de l'humain comprend de multiples polarités (corporelle, neurophysiologique, psychodynamique, la position de sujet, la liberté de l'individu, les relations sociales, l'histoire de vie,...). Ces polarités sont dans un continuum et forment un tout. Dans notre pratique, notre abord psychothérapeutique souvent pluridisciplinaire - a conscience de cette "pluri-polarité".

La formulation des items ci-après n'est nullement définitive, elle sera en transformation permanente durant la recherche pour déterminer ce qui est pertinent et ce qui ne l'est pas et, pour affiner les formulations et les exemples cliniques. Certains items sont corroborés par des données théoriques et cliniques de la recherche scientifique (Bediou et al., 2005 ; Bush et al., 2005 ; De Hert et al., 2001 ; Droit-Volet, 2000 et 2003 ; Ducato et al., 2005 ; Fossati et al., 2004 ; Georgieff, 2005 ; Henry et Gay, 2004 ; Lussier et Flessas, 2005 ; Mottron, 2003 ; Pliszka, 2003 ; State et al., 2002 ; Thomas et Willems, 2001 ; Van den Bergh, 2003 ; Wagner 2004 ; Zilbovicius, 2003). 
Voici, parmi d'autres, des exemples de notre repérage préliminaire :

\section{- Hypersensibilité à des bruits}

À propos d'un garçon de 3 ans et 3 mois, les parents expliquent que lorsqu'il était plus jeune, il ne supportait pas les bruits importants (avions, bouteilles jetées dans une bulle à verre, ...), et il avait peur quand il entendait le bruit de la pluie.

Une fillette de 5 ans conserve une hypersensibilité à certains bruits. Elle a peur de bruits forts, de machines bruyantes. (Par ailleurs, elle cherche une auto-stimulation auditive, et aime écouter ses musiques préférées avec un volume élevé ; nous constatons ici la coexistence d'une hypersensibilité et d'une sur-réactivité avec recherche de stimulations).

Une fillette de 18 mois a peur de certains bruits, par exemple, elle pleure si on se mouche, si elle entend mixer ou encore si on sonne à la porte. Elle demeure très peureuse.

\section{- "Débordé"}

Cette même petite fille, lors de certains pleurs, se montre débordée : elle hurle, devient agressive, s'agrippe avec rage et mord; la seule chose qui peut alors la calmer est de la mettre au lit.

Cette enfant est comme débordée par un trop plein d'informations ; elle réagit de façon craintive à des bruits intenses et est débordée par l'information interne que représentent ses affects lors des pleurs. Il faut alors la mettre au lit avec un maximum de calme pour éviter les stimuli afin de lui permettre de sortir de cet état de débordement et retrouver son contrôle.

\section{- Traitement de l'information auditivo-verbale}

Un garçon de 3 ans : lorsqu'il est en train de faire quelque chose, il ne fait plus attention à ce qu'on peut dire autour de lui. Il est sensible aux sons environnants, facilement distrait, lent à se situer lorsqu'il essaie anxieusement de comprendre ce qu'il entend. (Ceci peut correspondre à "l'extrême prépondérance de l'attention convergente" et à "un déficit de la capacité à distinguer le signal pertinent au sein de multiples stimuli auditifs").

Un garçon de 3 ans et demi, du fait d'une "sous-réactivité", écoute fugitivement, prête attention médiocrement et réagit peu à ce qu'on lui dit, cependant il réclame des bruits forts ou une musique intense.

Une fillette de 1 an et 8 mois est dérangée par des bruits nouveaux; un nouveau jouet qui fait de la musique peut la faire pleurer, elle a peur d'objets nouveaux qui produisent du bruit, il faut alors lui laisser quelques minutes pour qu'elle saisisse l'objet en l'apprivoisant elle-même. Elle a peur si elle entend du piano ou du saxophone (mais elle aime quand son père joue de la guitare). Elle a peur des voix graves, de même si on se mouche ou si on écrase une bouteille en plastique. Par ailleurs, elle peut rechercher la stimulation auditive, elle aime la musique. À l'âge de 2 ans et 1 mois l'hypersensibilité à certains bruits est moins intense ; toutefois, lorsqu'elle est occupée à une activité (par exemple crayonner) le bruit perturbe son attention et elle peut se sentir "débordée" (ceci peut correspondre à une "hypersensibilité" avec évitement de certains sons et à "un déficit de la capacité à distinguer le signal pertinent au sein de multiples stimuli auditifs").

\section{- Sous-réactivité visuelle}

À propos d'un garçon de 3 ans, (pour lequel les investigations neurologiques sont demeurées négatives), les parents rapportent que, jusqu'à l'âge de 2 ans, il avait parfois le regard fixe, dans le vide, «il partait dans la lune, il était ailleurs » et ce dans n'importe quelle circonstance. Par exemple, il conservait parfois le regard fixe en voiture même quand on essayait activement de l'accrocher ; mais à d'autres moments, il était pleinement réactif.

\section{- Hypersensibilité à des lumières avec évitement d'apports sensoriels visuels}

Un garçon de 3 ans et 5 mois fronce habituellement les yeux devant une "lampe néon" ou devant des rayons de soleil. Particulièrement lors de la première année de vie, mais encore actuellement, il est peu interactif par le média du canal visuel et est en pleine interaction par le canal auditif, souvent sans regarder son interlocuteur. Les examens neurologiques ont révélé une "sous-réactivité visuelle" due à un "visual maturation delay" : retard de maturation des voies visuelles centrales, s'améliorant partiellement au fil du temps. Il y a donc coexistence d'une hypersensibilité à des lumières et d'une sous-réactivité visuelle.

Depuis la naissance, un garçon de 2 ans et 8 mois a besoin du noir complet pour dormir. Les parents ont dû installer un système d'occultation complète des fenêtres de sa chambre.

\section{- Déficit du traitement de l'information visuo-spatiale}

Ce même garçon, voyant un objet sur une étagère, a eu peur et s'est mit à crier dans un état de panique : « il y a un monstre », comme d'un objet non identifié par lui. C'était une marionnette. Il a habituellement peur de tous les masques. Ceci reflète une difficulté du décodage des visages, tant au niveau de la forme que du contenu affectif, et de l'intégration de ces deux aspects, (les informations correspondant aux formes sont acheminées, depuis le cortex visuel jusqu'au lobe temporal, où l'image est reconstituée et identifiée de façon neutre, l'expression affective est elle décodée au niveau du système limbique).

Une fillette de 4 ans et demi peut avoir une "peur bleue" en voyant des clowns, des gens déguisés, maquillés (difficulté à décrypter la signification des visages très caricaturaux, difficulté à mettre en lien la reconnaissance de la forme du visage et la reconnaissance du sentiment exprimé par le même visage). 
Un garçon de 4 ans et 8 mois, qui a le même type de difficultés, met toujours ses mains sur la figure de sa petite soeur et a tendance à mettre ses mains devant le visage d'autres enfants et adultes.

Une fillette de 5 ans et 1 mois est dans la relation et l'échange ; pourtant, depuis longtemps, la famille et l'institutrice constatent qu'elle a tendance "à se faire une bulle", "à se renfermer sur elle-même", et qu'elle ne s'ouvre pas facilement aux autres. On a observé que lors d'une fête de carnaval elle était très impressionnée par les enfants qui portaient des masques.

\section{- Déficit de la capacité à discriminer les sentiments sur les visages}

Un garçon de 5 ans et 1 mois, en jouant avec des marionnettes stylisées en bois, fait un très grand nombre d'erreurs pour déterminer le sexe des marionnettes. En lui demandant de reconnaître une série de 10 affects, (chacun représenté de façon très expressive sur le personnage principal d'une scène développée en trois séquences d'une bande dessinée), il fait un nombre d'erreurs très important quant à la reconnaissance et l'interprétation du sentiment exprimé par les traits du personnage et la scène. Par ailleurs cet enfant a pourtant une bonne capacité de langage. Les parents notent que lorsqu'il était petit, il avait peur d'un clown qui se trouvait dans la chambre des parents ; il avait également peur d'une dame qui faisait le clown.

Un garçon de 3 ans et 2 mois, lors d'émissions TV, lorsqu'il voit des personnages avec des traits de visages caricaturaux (nez pointu, crochu,...), court et va se cacher derrière un fauteuil.

- Recherche de champs visuels et d'angles de vue variés et complexes

Ce même garçon regarde parfois les objets et jouets de tout près, les plaçant devant un oeil, en changeant l'angle de vision.

Une fillette de 3 ans et demi, systématiquement lorsqu'elle joue avec une maison Fisher-Price, regarde le champ visuel obtenu en accolant un oeil devant chaque fenêtre ou devant la porte ouverte.

\section{- Évaluation visuelle des mouvements des objets}

Un garçon de 2 ans et 8 mois (cité plus haut), depuis tout petit, en voiture, est systématiquement en insécurité en voyant les autres voitures, et peut parfois s'écrier : «attention aux voitures ». Certains enfants ont des difficultés à évaluer l'espace et la distance et à situer la trajectoire visuelle des objets. Cette difficulté peut provoquer un stress considérable.

\section{- Évaluation auditive des mouvements des objets}

La difficulté d'évaluer la trajectoire d'un objet à partir de l'audition peut exister. Entendant un objet se déplaçant bruyamment, l'enfant ne peut pas déterminer si cet objet se rapproche ou s'éloigne de lui. Un garçon et une fille de 3 ans présentent les mêmes réactions : dans la rue, lorsqu'ils entendent une voiture passer - sans la voir -, ils ont tendance à se coller à la personne qui les accompagne ou à longer les façades des maisons.

\section{- Évaluation visuelle des messages et signaux analogiques}

Une fillette de 2 ans et 10 mois est effrayée lorsque quelqu'un s'approche subitement d'elle, mais elle est à l'aise lorsque c'est elle qui prend l'initiative d'un contact physique en cherchant la relation ou les câlins. Cette difficulté est d'autant plus manifeste si la personne qui s'approche d'elle est expressive par ses attitudes, gestes et expressions analogiques. Il y a comme une déformation du décodage visuel des messages de l'expression analogique, tant au niveau de leurs messages formels que de leurs messages affectifs. Cette même enfant répond généralement aux questions relatives à son jeu, mais lorsqu'on la sollicite par des signaux et des gestes, elle n'interagit pas spontanément, ne comprenant pas les signaux et les gestes à moins qu'ils ne soient simultanément verbalisés.

Un garçon de 4 ans et 8 mois, sans antécédent de maltraitance, réagit parfois, lorsqu'on s'approche de lui, par exemple pour le prendre dans les bras, comme si on allait le frapper. (Cet enfant présente d'autres difficultés du décryptage de l'information visuo-spatiale).

Dans une famille où règne une bonne entente parentale, une fillette de 2 ans et 11 mois est vite impressionnée par certaines situations analogiques faites d'interactions et de mouvements entre les personnes. Lorsque ses parents interagissent entre eux, elle peut par exemple crier à sa mère : "toi, pas faire mal à papa !", bien qu'il n'y ait aucune dispute mais des interactions de jeux.

\section{- Difficultés au niveau de la sphère buccale}

Un garçon de 3 ans et 1 mois et une fille de 4 ans, avec retard du développement du langage, présentent les mêmes symptômes :

1. Faible tonicité musculaire et manque de coordination au niveau de la sphère buccale :

Ils ont tendance à baver et ils ont une très mauvaise articulation des mots.

2. Hypersensibilité tactile de la sphère gustative :

Ils évitent les aliments d'une certaine consistance. La fille prend des biberons en soirée.

3. Déficit de la capacité à organiser l'articulation :

Ils ont des difficultés à reproduire correctement les phonèmes des mots ; les mots sont raccourcis. (Ils ont aussi une difficulté à élaborer des phrases). 
4. Hypersensibilité du réflexe nauséeux avec hypotonie et hypersensibilité tactile de la sphère buccale :

Un autre garçon de 4 ans et 8 mois ne supporte pas les aliments consistants et tout ce qu'il mange doit être mixé ou écrasé. Il refuse tous les morceaux qu'il doit mordre et mâcher, même les "bonbons". Il a des difficultés à souffler dans l'eau du bain, à boire à la paille. L'articulation vocale est perturbée et il présente du sigmatisme (difficulté de prononcer la lettre "s"). On a constaté une hypersensibilité du réflexe nauséeux (qui a été rééduquée entre autre par l'introduction de plus en plus importante de la brosse à dents dans la bouche). L'O.R.L. a constaté des difficultés de déglutition. On note que bébé, il ne portait aucun jouet en bouche.

- Hypersensibilité gustative

Ce même garçon refuse des boissons variées telles que coca, jus de fruits... Il ne boit que de l'eau. Il refuse de goûter des aliments nouveaux.

Un garçon de 4 ans et 4 mois a des goûts sélectifs : il continue à refuser les bonbons, les sucreries, le coca, ..., il n'accepte que les pâtes bolognaises et le riz, il préfère les nourritures mixées et prend toujours un biberon le soir.

\section{- Insécurité gravitationnelle}

Un garçon de 3 ans et 1 mois a peur des situations qui mettent son corps dans des changements rapides de mouvements cinétiques tels que : être poussé en vélo, en balançoire, en carrousel, "être jeté en l'air",...

- État interne "hypo-énergétique"

Un garçon se réveillait toutes les 3 heures jusqu'à l'âge de 3 mois, puis il est devenu un grand dormeur. Jusqu'à l'âge de 2 ans, il ne se réveillait que deux à trois fois par jour en pleurant beaucoup et pour le reste il dormait quasiment 21 heures par jour (selon ses parents). Il a marché à 26 mois. Actuellement il a 5 ans, est atteint d'une maladie de Recklinghausen et, présente une déficience intellectuelle.

- État interne "hyper-énergétique"

Un garçon était un bébé très agréable, il a vite réduit la durée des siestes, vers 2 ans il a réduit le nombre d'heures de sommeil. Vers 2 ans et demi l'enfant reste actif après la mise au lit, il ne s'endort qu'à 23 h 30 , et il se lève à $7 \mathrm{~h} 30$ tout sourire et en "superforme".

Une fillette de 3 ans et 5 mois est habituellement mise au lit vers 20 h 30 mais elle reste d'abord animée avec des cris, bruits, et elle rit. Elle ne s'endort qu'après une demi-heure. Elle se réveille le matin en pleine forme, parfois déjà à $6 \mathrm{~h} \mathrm{30}$. Ses siestes sont plutôt rares. Elle joue beaucoup avec ses frères, aime être chatouillée. Elle arrache le papier peint, le tapis de sa chambre, prend n'importe quel objet dans la bouche (c'està-dire qu'elle fait du n'importe quoi avec n'importe quoi). (Ce qui se retrouve dans les symptômes présents dans des tempéraments maniaques).

- Perception des affects (sentiments et émotions)

1. Déficit de la capacité à percevoir sa propre affectivité (sentiments et émotions) et donc de la capacité à l'exprimer (alexithymie) :

Un garçon de 4 ans et 8 mois ne peut quasiment rien exprimer des événements qu'il a vécu la journée (avoir eu mal à la tête ou un affect important qu'il vient de vivre à l'école,...). Une émotion de mécontentement est contenue ou exprimée très rapidement avec distance ou encore avec un "humour" mal à propos. Peu de registres émotionnels sont exprimés.

2. Déficit de la capacité de la "perception intersubjective" ou "perception empathique" : Déficit de la capacité à percevoir l'affectivité profonde et unifiée des interlocuteurs, c'est-à-dire "l'état d'âme" de ses proches, et donc, déficit de la capacité à entrer dans un rapport "dialogual" profond et en congruence avec ses interlocuteurs :

D'une façon générale, ce même garçon se montre inadéquat dans les relations, il a des comportements et paroles "hors propos". Il doit s'imposer dans les relations. Il a une mauvaise appréciation de ce qui est en jeu dans la relation intersubjective. On peut repérer clairement qu'il a une mauvaise discrimination des sentiments sur les visages.

Un garçon de 3 ans et 4 mois ne supporte pas les remarques, est susceptible. Il s'entend très bien avec son copain et voisin, puis projette sur lui une bouteille de coca sans raison apparente. Il peut mordre et être agressif envers sa fratrie et les élèves de sa classe, ce qui rend les enfants méfiants à son égard et exaspère sa mère qui l'a une fois mordu "pour qu'il comprenne ce que ça fait". Il fait mal au chien de la famille. À d'autres moments, il est dans des interactions positives. Il a comme une "cécité" qui le rend incapable de voir ce que peuvent vivre ses interlocuteurs dans leur "monde interne".

- Organisation émotionnelle

1. Hyperlabilité émotionnelle : 
Une fillette de 4 ans et demi ne parvient pas à gérer l'intensité de ses émotions. Leurs modulations sont brusques et intenses ; elle présente des passages émotionnels en "tout ou rien". Lorsqu'elle est exubérante elle ne parvient plus à contrôler ses mouvements et peut ainsi être inadéquate envers ses pairs.

2. Modification de la tonalité émotionnelle prédominante habituelle (avec changement du "niveau interne d'énergie") :

Une fillette de 4 ans et 8 mois a de façon habituelle une tonalité émotionnelle prédominante énergique, espiègle, dominante, fonceuse ; or, sans raison apparente, depuis 3 mois elle se montre inerte, inactive, sans idée pour s'occuper, n'ayant envie de rien, lente, comme fatiguée et triste. (Les examens somatiques étaient normaux, et la prise de $90 \mathrm{mg} / \mathrm{soir}$ de quétiapine lui a fait retrouver après 4 semaines sa tonalité émotionnelle habituelle). (Cette rupture correspondait à un état dépressif avec effondrement du niveau interne énergétique).

3. Bascule rapide d'une tonalité émotionnelle en son contraire (liée à un "miroir interne de l'image de soi" livrant une image déformée négative) :

Un garçon de 4 ans et 2 mois se voit dans une image négative surfaite. Lorsqu'on lui fait une remarque, aussitôt il va se cacher sous la table, ou parfois il fait "des vilains yeux menaçants". D'autre part, lorsqu'il est fâché, souvent il peut s'écrier : "méchante maman", "je ne t'aime pas",... Dès le départ, cet enfant a une mauvaise perception de lui-même et il a une prépondérance à se voir dans une image déformée. L'enfant a une tendance habituelle à se dévaloriser. Lorsqu'un interlocuteur lui fait une remarque, aussitôt l'enfant bascule dans une image de lui-même déformée et négative. Ainsi, l'enfant se voit dans une image bien plus négative que celle que ne lui prête l'interlocuteur (ce qui conduit l'enfant à se cacher de lui-même). D'autre part, prêtant à l'interlocuteur - dans une évidence pour l'enfant - un regard aussi négatif sur lui que l'enfant n'a de lui-même une image négative surfaite, l'enfant peut trouver ce regard méchant, ce qui le fait réagir de façon disproportionnée. Pourtant, en dehors de ces moments, sa relation aux autres est positive.

Une fillette de 4 ans et demi a souvent l'impression qu'on ne l'aime pas, elle se replie sur elle-même, ne veut pas parler, se braque, ou encore fait "le bébé". Sans raison proportionnée, elle peut basculer dans des états de colère, passant "de l'état d'ange à l'état de démon", en "on-off", (et se vivant lors de ce dernier état avec une image très négative d'elle-même).

4. Déficit de la capacité d'utiliser les affects comme faisant partie de l'interrelation. Déficit - au sein d'une interaction - de la capacité à "accorder" ses affects à partir des messages reçus de l'interlocuteur (par déficit de la "perception intersubjective" rendant déficiente l'inter-modulation réciproque) avec tendance à un dialogue "uni-directionnel" (qui tourne au "monologue") :

Un garçon de 4 ans et 4 mois se montre souvent très obstiné, têtu, "restant sur ses rails", avec des "idées fixes". Il s'obstine souvent à vouloir indéfiniment une même chose, quelles que soient les interventions à son égard. Cet enfant exprime peu de sentiments personnels. Il se met souvent à l'avant-plan et il faut qu'on s'intéresse à lui. Il voudrait que l'interlocuteur soit à sa disposition.

5. Débordement des possibilités de gestion du moi lorsque les émotions - quelle que soit leur naturedépassent un certain seuil d'intensité, avec par ailleurs un abaissement de ce seuil :

Une fillette de 3 ans et 6 mois a tendance à mettre brièvement les mains sur les oreilles lors de moments de charges émotionnelles, comme par exemple, lorsqu'elle est heureuse de rencontrer une personne, lorsqu'elle s'anime et devient exubérante au cours d'un jeu, quand elle n'a pas envie d'une situation,...

\section{- Manque d'efficience de "la jauge interne d'évaluation anticipative du temps"}

Un garçon de 5 ans et 5 mois présente un retard d'apparition des repères temporels, non pas dans leurs connaissances notionnelle et rationnelle, (car il est intelligent et maîtrise bien le vocabulaire), mais dans l'appréhension subjective du temps : "goûter la durée du temps" et "ressentir anticipativement ce que dure un certain laps de temps" lorsqu'on annonce une activité, par exemple pour l'après-midi, (ce qui est différent de la notion séquentielle du temps). Il a acquis les repères rationnels temporels, mais pourtant il demande toujours l'heure à ses parents, et répète la question de savoir pour quelle heure on viendra le rechercher (alors que rationnellement il connaît la réponse). Il a toujours besoin de savoir comment va se dérouler sa journée et il est perdu si on change le programme. Lorsqu'une activité ludique est prévue il demande souvent, par exemple : « c'est quand qu'on va au carrousel ? » bien qu'on lui a déjà donné la réponse à plusieurs reprises. Le support neurophysiologique de la jauge interne d'évaluation anticipative du temps semble déficient.

\section{- Troubles de l'attention - concentration}

Un garçon de 4 ans et 8 mois peut se disperser, il a une attention divergente. Dans un contexte donné il suffit qu'un mot entendu lui rappelle quelque chose pour qu'il raconte cette chose ou cette histoire, il passe "du coq à l'âne", et ainsi il peut avoir des comportements hors contexte. Il peut être facilement distrait, ne réagit pas toujours quand on lui demande quelque chose. Il a de la peine à suivre les consignes dans un groupe. Il va pouvoir réaliser les consignes et faire ce qui est demandé si l'institutrice est à coté de lui, mais dans le groupe, attablé avec 6 enfants, il ne parvient pas ou plus difficilement à les réaliser si l'institutrice est éloignée. 
- Hypersensibilité au contact de certaines zones corporelles avec évitement d'apports sensoriels

Un garçon de 3 ans et 5 mois n'aime pas quand on lui passe un pull ou quand on l'habille. Il a peur du passage du gant de toilette sur le visage.

Une fillette de 3 ans et 4 mois ne supporte pas de toucher certaines textures de tissus et semble très vite dérangée par des vêtements ou des souliers qui la serrent un peu.

Un garçon de 3 ans et 10 mois présente une hypersensibilité tactile au niveau des pieds alors que les autres parties du corps semblent normosensibles. Cela rend difficiles les soins de pédicurie alors que les soins des mains ne posent aucune difficulté.

- Hyper-réactivité au contact avec recherche d'apports sensoriels

Enfant qui cherche particulièrement à se faire câliner, chatouiller, caresser, serrer, "porter à cheval", "se faire attraper", ...

- Hyperactivité

Un garçon de 3 ans et 3 mois bouge beaucoup, aime se déplacer d'une pièce à l'autre. Le matin, son lit est défait et il est en travers du lit. Il passe fréquemment d'une chose à l'autre ... Il préfère les jeux moteurs : vélo, balançoires...

- Hypersensibilité au chaud avec évitement de la chaleur

Un garçon de 17 mois refuse systématiquement les aliments chauds, (alors qu'il accepte les mêmes aliments à température ambiante). Un garçon de 5 ans et 7 mois veut systématiquement prendre son bain avec de l'eau froide et refuse l'eau chaude.

- Hypersensibilité au froid avec évitement du froid

Une fillette de 3 ans et 1 mois ne supporte pas les glaçons à sucer : au contact du froid elle met aussitôt les mains sur les oreilles. De même, depuis qu'elle a des dents, elle refuse la crème glacée, dit que c'est bon mais qu'elle n'aime pas le froid.

- Sous-réactivité à la douleur

Un garçon de 4 ans et 6 mois présente une hyposensibilité à la douleur et on peut constater de nombreuses cicatrices sur son corps.

- Hypersensibilité de l'odorat

Ce même garçon présent une hypersensibilité de l'odorat et peut facilement reconnaître le propriétaire d'un pull-over à partir de son odeur.

- Hypotonie musculaire

- Incoordination des mouvements

- Motricité fine altérée

$\cdots$

\section{C) Élaboration d'un questionnaire pour une étape ultérieure de la recherche}

Ce travail préliminaire a permis d'élaborer un questionnaire, pour les enfants de 5 à 6 ans, à remplir par les parents. Ce questionnaire est rétroactif depuis la grossesse. Il cherche à mettre en évidence les Troubles de la Régulation et, nous souhaitons qu'il ait un intérêt pédopsychiatrique pour tout enfant présentant des difficultés. Ce questionnaire sera la base de la véritable recherche.

PERSPECTIVE DE RECHERCHE : COMPARAISON DES RÉSULTATS D'UN QUESTIONNAIRE ANAMNESTIQUE ENTRE UN GROUPE TÉMOIN ET UN GROUPE D'ENFANTS PRÉSENTANT UN DIAGNOSTIC DE TROUBLES DE LA RÉGULATION DU TRAITEMENT DES STIMULI SENSORIELS

L'objectif est de connaître les éléments anamnestiques qui sont significatifs dans la population présentant le diagnostic de Troubles de la Régulation du traitement des stimuli sensoriels. Le questionnaire réalisé par la recherche préliminaire sera rempli par des parents d'enfants de 5 ans répartis en deux échantillons, l'une présentant un diagnostic de Trouble de la Régulation, et l'autre étant un groupe témoin. Chaque population comprendra au moins 100 enfants. Dans l'analyse ultérieure nous chercherons à :

- Connaître la fréquence des réponses positives au questionnaire dans une population témoin.

- Connaître la fréquence des réponses positives au questionnaire dans une population présentant un diagnostic de Troubles de la Régulation.

- Comparer la fréquence de ces réponses entre les deux échantillons.

— En déduire les items significatifs du diagnostic de Troubles de la Régulation. 
Ceci permettra de mieux préciser la sémiologie significative des Troubles de la Régulation.

À partir de cette comparaison, nous établirons un nouveau questionnaire en ne retenant que les items les plus significatifs du diagnostic de Troubles de la Régulation. Ce nouveau questionnaire pourrait être un outil de dépistage, notamment dans les Services de Santé Mentale.

Alors que le Groupe de Travail Chargé de la Révision de la Classification Diagnostique (2005) a apprécié l'utilité des Troubles de la Régulation, les experts ont cependant souligné la nécessité de recherches cliniques futures pour mieux identifier les catégories utiles et le devenir au long terme de ces enfants (Fenichel, Emde et al., 2005). Notre recherche devrait permettre aussi de mieux en préciser les entités cliniques.

\section{LES APPLICATIONS RELATIONNELLES DE LA RECHERCHE}

\section{A) Perspective pour une meilleure compréhension de l'enchevêtrement des difficultés induites réciproquement par l'enfant et les parents}

L'observation de parents en relation à un enfant qui présente un Trouble de la Régulation, ouvre une recherche sur l'enchevêtrement des difficultés induites réciproquement par l'enfant et les parents. L'enfant qui présente un Trouble de la Régulation sera plus sensible aux facteurs de stress environnementaux et présentera des difficultés comportementales plus importantes que la moyenne des enfants dans la même situation. L'état de l'enfant pourra exacerber les sentiments des parents, d'autant que les difficultés de l'enfant ne seront pas reconnues comme étant en partie dues à sa sensibilité induite par le Trouble de la Régulation du traitement des stimuli sensoriels. Que du contraire, cela communiquera aux parents des sentiments de culpabilité et cela pourrait entrer en résonance avec leurs propres difficultés personnelles qui n'auraient peut-être pas été exacerbées si l'enfant n'avait pas présenté un Trouble de la Régulation du traitement des stimuli sensoriels (qui lui-même peut évoquer chez les parents des figures familières transgénérationnelles). Comme nous l'avons décrit plus haut, dans certains Troubles de la Régulation du traitement des stimuli sensoriels, certains enfants ont des difficultés à déchiffrer les signaux affectifs, qu'ils soient vocaux ou visuels. Ces enfants identifient mal les expressions de l'affectivité sur le visage et/ou ils identifient mal si les choses sont dites d'une voix gaie, triste ou irritée (Classification Études de cas, 2000). L'interaction sociale réciproque précoce entre le parent et l'enfant conduira le parent à trouver l'enfant insensible et difficile à réguler. La modulation des affects est importante pour développer leur tolérance mutuelle et leur accordage. On sait que les mères modulent et transforment les affects négatifs de leur bébé, de même qu'elles augmentent de façon positive le niveau optimal de leur éveil, en exagérant leurs propres expressions faciales et vocales. Cependant, pour que cela soit efficace, l'enfant doit être capable de capter des signaux affectifs communiqués par la voix, par le visage, par les gestes ou de façon interactive. Sinon, l'enfant n'éprouvera probablement qu'une émotion et une tension négative plus forte, auxquelles il aura des difficultés à faire face. Ces nuances peuvent être très précieuses pour un thérapeute et, un abord thérapeutique peut tenir compte à la fois de composantes constitutionnelles et relationnelles dans un continuum.

\section{B) Perspective de métaphores thérapeutiques}

Lors d'un premier rendez-vous avec les parents, le regard du thérapeute sur l'enfant peut accroître une "relation thérapeutique". Par exemple, si des parents viennent avec un enfant "du spectre autistique", leur demander de faire un portrait complet de leur enfant, non seulement des aspects "problématiques" mais de tous les aspects, y compris des bons cotés et des compétences, permet aux parents de décrire leur enfant dans toutes ses réalités, et au thérapeute de s'ouvrir à leurs regards. Ainsi les parents peuvent se sentir entendus, respectés, ils peuvent exprimer leurs préoccupations et leurs traductions dans le quotidien. En un second temps, le thérapeute pourra restituer des explications constitutionnelles "neurophysiologiques" aux difficultés de l'enfant en reprenant toute une série de points de description donnés par les parents eux-mêmes. Il pourra indiquer l'utilité d'un abord thérapeutique et - si nécessaire - d'une médication spécifique à titre de traitement adjuvant. Cette nouvelle compréhension des difficultés de leur enfant - données aux parents à partir de leur propre description - va modifier leur contre-transfert à l'égard de l'enfant et ouvrir à nouveau une relation qu'éventuellement ce contretransfert mettait en difficulté. La reconnaissance des difficultés réelles de l'enfant communique aux parents une reconnaissance de ce qui est difficile pour eux et qui les met en difficulté. Notre expérience montre que cette reconnaissance des difficultés spécifiques de l'enfant - qui peut déculpabiliser les parents - leur permet alors d'aborder leurs propres difficultés affectives et relationnelles en lien avec leur histoire propre. Ainsi, il serait intéressant qu'une recherche évalue l'effet thérapeutique que peuvent apporter des métaphores explicatives des difficultés de l'enfant, composées à partir d'une compréhension d'une composante constitutionnelle de ces difficultés. 


\section{CONCLUSION}

Le diagnostic de Trouble de la Régulation du traitement des stimuli sensoriels est certainement le plus original et le plus innovateur de la Classification Diagnostique 0-3 ans. Ces enfants présentent des difficultés au niveau du traitement des stimuli sensoriels entrant et au niveau de la régulation des réponses qu'ils apportent à ces stimuli. La recherche ici présentée dans sa phase préliminaire, par une microanalyse qualitative, a cherché à attirer l'attention sur des nuances sémiologiques rencontrées chez des enfants présentant des Troubles de la Régulation. Entre autres, une attention toute particulière a été portée sur la dimension affective de l'enfant. La phase ultérieure de l'étude, actuellement en cours, cherchera à déterminer la pertinence et la fréquence des signes sémiologiques rencontrés. Pour ce faire, les résultats d'un questionnaire anamnestique seront comparés entre un groupe témoin et un groupe d'enfants présentant un Trouble de la Régulation. Nous espérons que cette clinique et cette recherche donneront accès à des observations qui peuvent contribuer à enrichir les échanges pluridisciplinaires et la pratique psychothérapeutique. Nous voudrions alimenter une réflexion permettant un meilleur discernement - dans un continuum - entre la part constitutionnelle, la part psychologique et la part relationnelle intervenant dans les difficultés de l'enfant.

\section{RÉFÉRENCES}

Bediou B., Krolak-Salmon P., Brunelin J., Saoud M., d'Amato Th. (2005), Déficit d'identification d'expression du visage dans la schizophrénie, L'Encéphale, 31, 2 ,251-252.

Bush G., Valera E., Seidman L.J. (2005), Functional Neuroimaging of Attention-Deficit/Hyperactivity Disorder: A Review and Suggeested Future Directions, Biol. Psychiatry, XX:XXX, 1-12.

DC: 0-3 Casebook (1997), by Zero to three/National Center for Infant, Toddlers and Families, Washington, DC. Trad. fr. Classification Diagnostique de 0 à 3 ans Études de cas (2000), Médecine et Hygiène, Genève.

De Hert M., Peuskens J., D’Haenens G., Hulselmans J., Janssen F., Meire I. (2001), Les limitations cognitives des patients schizophrènes: une caractéristique persistante de leur maladie, suppl. à Neurone, 6, 3, 1-10.

Droit-Volet S. (2000), Les différentes facettes du temps, Enfances et Psy, 13, 26-40.

Droit-Volet S., Wearden J. (2003), Les modèles d'horloge interne en psychologie du temps, L'année psychologique, 104, 617 654.

Ducato M.G., Thomas P., Boucart M. (2005), Capture attentionnelle dans la schizophrénie : effet de la charge attentionnelle, L'Encéphale, 31, 2, 254.

Fenichel E., Emde R.N., Egger H., Guédeney A., Wise B.K., Wright H.H. (2005), Clinique Présentation de la révision de la Classification Diagnostique 0-3 ans (DC:0-3R), Devenir, 17, 4, 347-360.

Fossati P., Hevenor S.J., Lepage M., Graham S.J., Grady C., Keightley M.L., Craik F., Mayberg H. (2004), Distributed self in episodic memory : Neural correlates of successful retrieval of self-encoded positive and negative personality traits, Neuroimage, 22, 1596-1604.

Georgieff N. (2005), Intérêts de la notion de «Théorie de l'esprit » pour la psychopathologie, Psychiatrie de l'enfant, 48, 2 , 341-371.

Guédeney N., Guédeney A., Danon G., Mintz A.S., Morales-Huet M., Rabouam C., Jacquemain F., Le Nestour A., Roujean S. (2002), À propos des systèmes de classification en psychiatrie du très jeune enfant : utilisation de la classification diagnostique Zero to three, Psychiatrie de l'enfant, 45, 2, 483-531.

Henry C., Gay C. (2004), État de la recherche dans les troubles bipolaires. Encyclopédie Orphanet janvier 2004.

Lussier F., Flessas J. (2005), Neuropsychologie de l'enfant. Troubles développementaux et de l'apprentissage, Ed. Dunod.

Mottron L. (2003), Une perception particulière, Dossier autisme, Cerveau et psycho, 4, 72-75.

Pliszka S.R. (2003), Neuroscience for the Mental Health Clinician, Ed. The Guilford Press, New York.

State R.C., Altshuler L.L., Frye M.A. (2002), Mania and Attention Deficit Hyperactivity Disorder in a Prepubertal Child : Diagnostic and Treatment Challenges, Am. J. Psychiatry, 159, 6, 918-924.

Thomas J.M., Harmon R.J. (1998), La classification diagnostique des troubles de la santé mentale et du développement de la première et de la petite enfance. Un système dynamique pour la connaissance et le traitement des nourrissons, des jeunes enfants et de leurs familles, Devenir, 10, 1, 35-50.

Thomas J., Willems G. (2001), Troubles de l'attention, impulsivité et hyperactivité chez l'enfant, Ed. Masson.

Van den Bergh W. (2003), L'EEG-biofeedback dans l'ADHA, Neurodynamique de l'ADHD et application thérapeutique, L'agenda Pédiatrie, 36, 4-6.

Wagner K.D. (2004), Diagnosis and Treatment of Bipolar Disorder in Children and Adolescents, J Clin Psychiatry, 65, 15, 30-34.

ZERO TO THREE: Diagnostic Classification of Mental Health and Developmental Disorders of Infancy and Early Childhood (DC:0-3), (1994), Washington, DC. Trad. fr. Classification Diagnostique de 0 à 3 ans (1998), Médecine et Hygiène, Genève. 
ZERO TO THREE: Diagnostic Classification of Mental Health and Developmental Disorders of Infancy and Early Childhood (DC:0-3R), Revised Edition (2005), ZERO TO THREE Press, Washington, DC.

Zilbovicius M. (2003), Imagerie cérébrale et autisme infantile, Cerveau et psycho, 4, 80-83.

Dr Scholl Jean-Marc

2006

Service de Santé Mentale

Rue des Déportés, 30

4800 Verviers

Belgique

Jean-Marc.Scholl@chu.ulg.ac.be 